See discussions, stats, and author profiles for this publication at: https://www.researchgate.net/publication/306007992

\title{
Sensing of damage and substrate stress in concrete using electro-mechanical impedance measurements of bonded PZT patches
}

Article in Smart Materials and Structures · August 2016

DOI: 10.1088/0964-1726/25/9/095011

2 authors:

Arun Narayanan

Indian Institute of Technology Hyderabad 10 PUBLICATIONS 27 CITATIONS

SEE PROFILE
Kolluru Subramaniam

Indian Institute of Technology Hyderabad 130 PUBLICATIONS $\mathbf{1 , 4 8 1}$ CITATIONS

SEE PROFILE

Some of the authors of this publication are also working on these related projects:

Damage Assesment of Concrete Elements using PZT Based Sensors View project

Seismic Assessment of Masonry Structures View project 


\title{
Sensing of damage and substrate stress in concrete using electro-mechanical impedance measurements of bonded PZT patches
}

\author{
Arun Narayanan and Kolluru V L Subramaniam \\ Department of Civil Engineering, Indian Institute of Technology Hyderabad, Hyderabad, TS 502285, India \\ E-mail: kvls@iith.ac.in
}

Received 29 January 2016, revised 11 June 2016

Accepted for publication 16 June 2016

Published DD MM 2016

\begin{abstract}
The influence of stress and induced damage in concrete on the electro-mechanical (EM) impedance response of bonded PZT patches is evaluated for applied compressive loading. Full field displacements obtained from digital image correlation are used to evaluate the level of stress-induced damage in concrete. Stress in the substrate produces an imposed strain on the PZT. A change in the imposed strain produces a rightward frequency shift and an increase in the amplitude of the resonant peak in the EM conductance spectrum of the PZT. An increase in the substrate compliance produces a decrease in the resonant frequency and an increase in the amplitude of the resonant peak. Changes in the resonant peak in the conductance spectrum induced by increasing substrate stress are of a significant magnitude when compared with the changes induced by damage. In the early stages of damage associated with distributed microcracking, the counteracting influences of increasing level of damage and increasing stress on the resonant peak result in no shift in frequency for measurements under applied load. There is however an increase in the amplitude of the resonance peak. When the applied stress is removed, there is a net decrease in frequency resulting from damage in the form of distributed microcracks. Measures of changes in the resonant peak based on root mean square deviation (RMSD), do not show any observable change when measurements are performed under applied loading. There is a consistent increase in RMSD values and frequency shift with increasing damage when the applied stress is removed. The centroidal measure of the normalized frequency spectrum reflects changes in substrate stress. At higher applied stress levels, there is a nonlinear increase in damage, leading to localization and cracking. The influence of damage is dominant in this region and significant changes are obtained in the RMSD values in both loaded and unloaded conditions.
\end{abstract}

Keywords: electro-mechanical impedance, PZT, digital image correlation, stress, damage, conductance, compliance

SQ1 (Some figures may appear in colour only in the online journal)

\section{Introduction}

Signs of distress in concrete are often associated with visible cracking. Since concrete is a brittle material, which is weak in tension, cracking is the manifestation of damage in the material which results from tensile stress in the material. Stress induced damage in concrete could result from load application or due to effects such as restrained shrinkage and thermal contraction. Damage initiation is stress-induced and takes place in the form of distributed microcracks, which eventually coalesce to form localized cracks. By the time surface manifestation in the form of visible cracking appears there may be 
significant degradation of the capacity of the structure. Early detection of damage is essential to initiate early intervention measures, which can effectively increase the service life of a structure. Methods to detect incipient damage in the form of microcracks are required to provide effective methods of monitoring structural health and service life performance of structures.

Impedance-based measurements of lead zirconate titanate (PZT) patches bonded to a concrete substrate provide an effective way for monitoring incipient damage in the material. Studies of damage in concrete using impedance-based measurements of PZTs have been conducted using embedded defects and machine cuts (Tseng and Wang 2004, Lim et al 2006, Dongyu et al 2010, Wang et al 2013). In these studies, EM impedance measurements were conducted for increasing level of artificial damage. The influence of artificial damage on the mechanical impedance experienced by the PZT is not identical to that of load-induced damage (Narayanan and Subramaniam 2016). In concrete structures, the evolution of material damage from distributed microcracks to localization in the form of visible cracking is also associated with a change in the level of strain in the material medium and an accompanied increase in material compliance (Chandra and Shah 1968, Gopalaratnam and Shah 1985). Experimental studies on the influence of load induced damage on the response of bonded PZT have also been reported (Park et al 2000, Soh et al 2000, Yang and Hu 2008). In these tests, the response of PZTs were continuously monitored for monotonically increasing load. The PZT is placed in a stress field and therefore the measured response of the PZT in such experiments includes the influence of the stress in the substrate, in addition to the effect of increase in the level of damage in the material. The influence of load induced damage in compression without the influence of applied stress was investigated using specimens unloaded from a specific load level. In measurements performed in the unloaded state, the level of material damage was evaluated using change in the equivalent stiffness extracted from identified equivalent system model, which is derived from the drive point mechanical impedance within a narrow frequency range from EM impedance response of PZT (Soh and Bhalla 2005). In the unloaded state, microcrack damage in concrete was shown to be associated with increases in the level of damping and the material compliance. The combined influence of the two effects produces a decrease in the frequencies of the resonant peaks in the electro-mechanical (EM) impedance measurement of a PZT bonded to a concrete substrate (Narayanan and Subramaniam 2016).

Some studies on the influence of stress in the substrate on the response of a bonded PZT have been reported. Most of these studies have been performed on metallic substrates using small specimen and the influence of stress was evaluated using structural modes in the EM impedance response of the PZT. For increasing strain in the substrate, frequency shifts and amplitude changes in the structural resonant modes have been reported (Ong et al 2002, Annamdas et al 2007, Lim and Soh 2012, 2013). The susceptance part of the measured admittance spectrum associated with the structural modes obtained from an EM impedance measurement of the bonded PZT is shown to be more sensitive than real part of the admittance for monitoring stress in the substrate (Annamdas et al 2007). The effect of stress in the substrate on the PZT conductance signature were studied using Aluminium specimen and a compensation technique using effective frequency shift by cross-correlation analysis was applied to conductance spectrum to compensate the effect of loading (Lim and Soh 2013). The individual effect of load and damage on the structural peaks were decoupled by considering the influence of stress obtained from a pristine specimen as a correction for the specimen with damage in the form of an artificial cut at the corresponding stress level. Decoupling approach is not directly possible in concrete structures, since an increasing stress level in the substrate is often associated with an increase in the level of damage. Further, considering the energy requirements for exciting structural modes, the use of these modes in concrete elements is not be feasible.

Embedded PZT smart aggregates were used to observe the effect of stress at various levels by observing the changes in embedded PZT resonance peaks (Dongyu et al 2014, Saravanan et al 2015). It has been reported that the embedded smart aggregates are insensitive to the stress since the local stress in the material is different from the applied stress field. There were no systematic trends observed in the impedance resonance peak and the interpretations were very subjective. In embedded smart aggregates, there are several intervening effects which do not allow the delineation of influence of stress and damage in the substrate.

In concrete structures, an increase in the applied stress is often linked with an increment in the level of material damage. An increase in the level of damage produces a change in the substrate compliance. At an applied stress level, an increase in substrate compliance results in an increase in the strain. Therefore, a PZT coupled to a concrete substrate potentially experiences a change in imposed strain due to a changes applied stress. The resulting change in the substrate compliance and material damping produced by an increment in the damage influence the EM impedance response of a bonded PZT. Practical application of the impedance-based measurements to detect incipient damage in concrete structures requires an understanding of the influence of the level of damage and substrate stress on the response of a PZT bonded to the substrate. The use of EM impedance-based monitoring of concrete structures requires identifying changes in the impedance signature due to an increment in the level of damage and a change in the applied stress. Further, the influence of both phenomena on the observed impedance response of a bonded PZT patch need to be separated. In this paper an experimental investigation of concrete subjected to incremental levels of damage and stress are reported. The level of damage in concrete is increased in a controlled manner using progressively incremental loading. The level of substrate strain associated with applied stress and material damage are obtained using digital image correlation (DIC). The influence of damage and strain in the concrete substrate 
Table 1. Properties of materials

\begin{tabular}{lcccc}
\hline Type & Avg. compressive Strength (MPa) & $\begin{array}{c}\text { Avg. Young's Mod- } \\
\text { ulus (GPa) }\end{array}$ & Density $(\rho)\left(\mathrm{kg} \mathrm{m}^{-3}\right)$ & Poisons ratio $(v)$ \\
\hline High strength concrete & 75 (Std. dev. 3.35) & 44 & 2370 & 0.2 \\
Normal strength concrete & 49 (std. dev. 3) & 35 & 2300 & 0.2 \\
Epoxy & - & 2 & 1400 & 0.36 \\
\hline
\end{tabular}

on the EM impedance response of a bonded PZT are identified.

\section{Background}

In a piezoelectric material, the application of an electrical field results in mechanical strain in the material due to the coupled electro-mechanical constitutive relations. The constitutive

Q1 relation for a PZT is given as (IEEE 1986, Sirohi and Chopra 2000)

$$
\begin{aligned}
& D_{i}=e^{\sigma}{ }_{i j} E_{j}+d^{d}{ }_{i m} \sigma_{m}, \\
& \varepsilon_{k}=d^{c}{ }_{j k} E_{j}+s^{E}{ }_{k m} \sigma_{m},
\end{aligned}
$$

where $D_{i}$ is the electric displacement, $\varepsilon_{k}$ the mechanical strain, $E_{j}$ the electric field and $\sigma_{m}$ the mechanical stress, $e^{\sigma}{ }_{i j}$ is the complex electric permittivity of the PZT material at constant stress, $d^{c}{ }_{j k}$ and $d^{d}{ }_{i m}$ are the piezoelectric strain coefficients and $s^{E}{ }_{k m}$ the complex elastic compliance at constant electric field. The superscripts $c$ and $d$ have been added to differentiate between the converse and direct piezoelectric effects. The superscripts $\sigma$ and $E$ indicate that the quantity is measured at constant stress and constant electric field, respectively. From the constitutive relation of piezoelectric material, it can be seen that there is a coupling between electrical displacement and applied stress through piezoelectric strain coefficient. Change in the applied stress will reflect a change in electrical displacement of the PZT, and thus the conductance of the PZT.

For a PZT patch attached to a substrate subjected to an applied electrical input, the motion of the interface subjected to continuity conditions is governed by the combined mechanical impedance of the structure and the PZT. The constrained motion in turn produces a change in the measured electrical impedance. The first systematic attempt to derive the electrical impedance of the PZT which is mechanically connected to a structure using a 1D idealization of the system was developed by Liang et al (1994). Subsequent improvements in modeling the PZT response have included the effective 1D model of the PZT and varying levels of idealization of the structural impedance (Bhalla et al 2004, Xu and Liu 2002, Yang et al 2005). Most of the available analytical solutions are applicable for 1 or 2D idealizations of the PZT, substrate or both. Typically, the electrical admittance of the PZT patch $(\bar{Y})$ for a given electrical input at a frequency can be represented as

$$
\bar{Y}=\bar{Y}\left(Z_{A}, Z_{S}, \omega, l_{i}, E\right),
$$

Table 2. Properties of PZT.

\begin{tabular}{ll}
\hline Density $\rho_{p}\left(\mathrm{~kg} \mathrm{~m}^{-3}\right)$ & 7700 \\
Young's modulus (GPa) & 62.5 \\
Poisson's ratio, $v_{p}$ & 0.35 \\
Mechanical quality factor $\left(Q_{m}\right)$ & 75 \\
Piezoelectric strain coefficients, $d 31, d 32\left(\times 10^{-12} \mathrm{~m} \mathrm{v}^{-1}\right)$ & -195 \\
Piezoelectric charge coefficient, $d 33\left(\times 10^{-12} \mathrm{~m} \mathrm{v}^{-1}\right)$ & 450 \\
Dielectric loss factor, $\delta$ & 0.02 \\
Relative permittivity & 1700 \\
\hline
\end{tabular}

where $Z_{A}$ And $Z_{S}$ are the mechanical impedance of the PZT and substrate respectively. $l_{i}$, represent the dimensions of patch and $E$ is the electric field applied for actuation. Such equations have been derived assuming the mean strain in the PZT is zero.

Damage in the material produces a change in its mechanical compliance, and the associated change in the substrate mechanical impedance $\left(Z_{S}\right)$ results in a change the electrical admittance of the PZT patch $(\bar{Y})$. Changes in substrate strain due to increasing damage and applied stress would result in an imposed strain on the PZT. Considering the coupled electro-mechanical constitutive relations of the PZT (given in equations (1) and (2)), the imposed strain on the PZT would also produce a change in its EM impedance signature.

\section{Materials and methods}

An experimental program was conducted for measuring the electrical impedance of PZT patches attached to concrete cubes made with two different grades of concrete. $150 \mathrm{~mm}$ concrete cubes were used in the test program. Three cubes from each concrete batch were tested to determine the elastic properties and compressive strength of concrete. The material properties of the normal strength concrete (NSC) and high strength concrete (HSC) are listed in table 1. The material properties of the PZT are given in table 2 .

Electrical impedance measurements were performed on a PZT patch bonded to a concrete substrate for different levels of compressive stress in the substrate and compression stressinduced damage in the material. The EM impedance measurement consisted of measuring the frequency dependent electrical admittance of the bonded PZT. The frequency dependent conductance spectrum derived from the electrical admittance of the PZT was used in analysis. A square PZT patch of $1 \mathrm{~mm}$ thickness, and $20 \mathrm{~mm}$ length were attached to the center of one of the face of $150 \mathrm{~mm}$ concrete cube for 


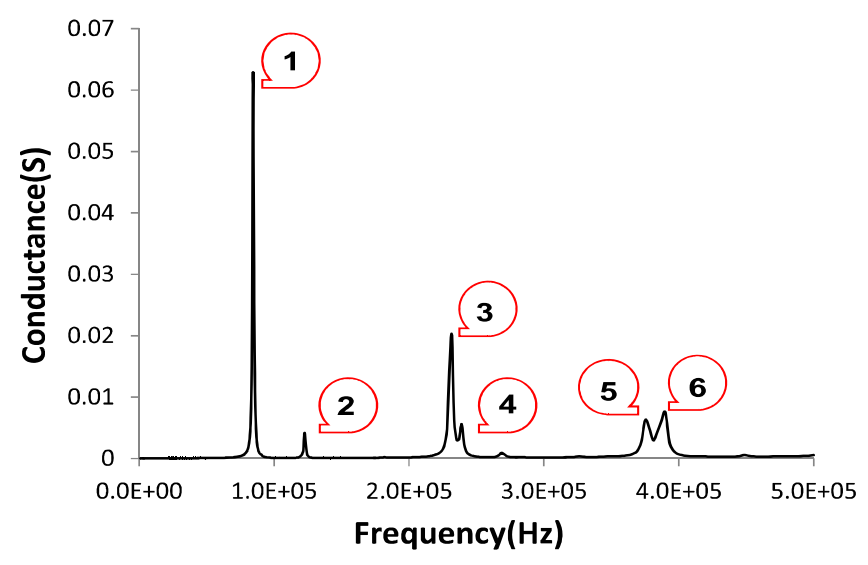

(a)

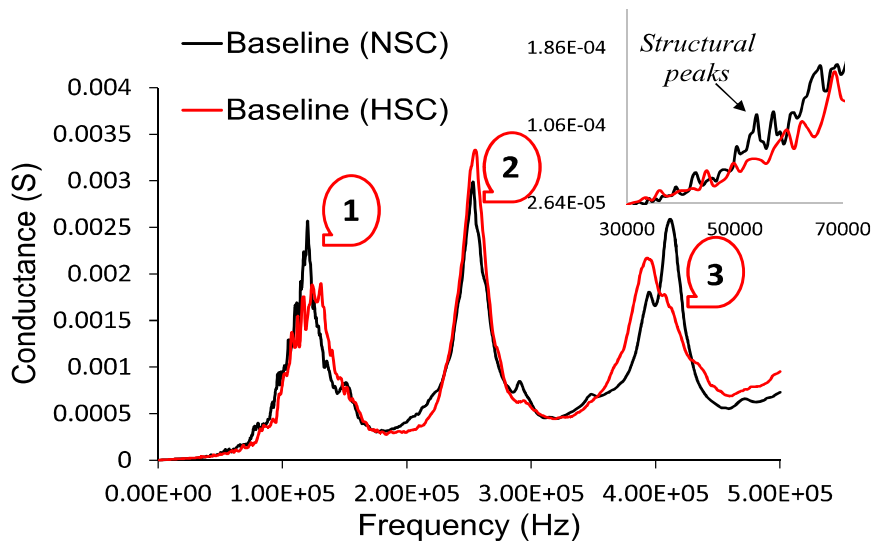

(b)

Figure 1. The conductance spectrum obtained from the EM impedance measurement: (a) free PZT; and (b) PZT bonded to concrete cubes.

experimental study (figure 2(a)). A two-component epoxy adhesive was used to bond the PZT patch to concrete cube. Electrical admittance signatures were collected from the PZT patches in the free-state before attaching them to concrete cubes. In a typical admittance measurement, the frequency was varied between $1 \mathrm{kHz}$ and $0.5 \mathrm{MHz}$ at an applied voltage of $1 \mathrm{~V}$ and data was collected at 800 discrete frequencies. Average of five measurements were collected to minimize the influence of random noise. The admittance signature of the PZT patch was collected in the different loaded and unloaded states by subjecting the concrete cube to cyclic compressive loading. The poled direction of the PZT was kept normal to direction of loading.

The conductance signatures derived from the electrical admittance measurements on the PZT patches bonded to NSC and HSC cubes are shown in figure 1(b). The conductance signature of the PZT in the free-state is also shown in the figure 1(a) with its resonant modes marked for reference. The PZT resonant modes are identified by the peaks in the response of the free PZT. Comparing the responses of the free and the bonded PZTs, it can be seen that the dominant PZT resonant modes are clearly identified in the response of the bonded PZT at frequencies close to the resonant frequencies of the free PZT. In the measured conductance response of the bonded PZT, the first, third, fourth and fifth resonant modes of PZT are prominently identified with peaks in the spectrum. The first and second peaks in the responses of the bonded PZT are identified with the first and third resonant peaks of the free PZT. The third resonant peak in the response of the bonded PZT is associated with the fifth and sixth resonance modes of the free PZT. The second and fourth resonance modes of the free PZT are suppressed in the impedance response of the bonded PZT. In the EM response of bonded PZT there is an increasing trend in the background conductance with increasing frequency.

The low frequency response contain closely spaced peak (shown in the inset in figure 1(b)) and are identified with the structural modes of the cube. The structural modes in the response of the bonded PZT are found to be overlapping with the first resonance mode of the PZT in the conductance spectrum of the bonded PZT. The first resonant peak of the bonded PZT is not well defined in HSC when compared with NSC cube because of the significant overlap of structural modes. The higher material stiffness of HSC produces structural resonant modes at higher frequencies than the NSC. The third peak in the bonded PZT response is produced by two closely spaced modes and the relative magnitudes of the two modes are different for the two grades of concrete; while in the stiffer HSC, the lower frequency mode has higher amplitude, the higher frequency mode has relatively higher magnitude in NSC. Objective identification and comparative evaluation of the influence of damage, free from the influence of boundary effects and baseline material compliance in HSC and NSC would not be possible using the first and third peaks in the conductance spectra of bonded PZT. The second peak in the impedance response of the bonded PZT, identified with the third resonant mode of the free PZT was selected for evaluation since it is consistently well defined in both HSC ad NSC cubes and is associated with a single PZT mode. The influence of substrate impedance on the response of second peak in the impedance response of bonded PZT is obtained by comparing with the third resonant peak of free PZT. The center frequency of the third peak of the free PZT is $236 \mathrm{kHz}$ and the second peak in the conductance spectrum of the bonded PZT is centered on $256 \mathrm{kHz}$. Considering the free PZT to be the limit case of a PZT attached to a substrate material with no mechanical impedance, an increase in material impedance is associated with a decrease in amplitude and an increase in the frequency of the resonant peak.

In the test program, the compressive stress in the substrate was incremented in a controlled manner using a computer controlled servo-hydraulic testing machine. To ensure uniform contact with the platens of the test machine, the concrete cubes were levelled using a capping compound. The concrete cubes were subjected to cyclic compressive loading where the load amplitude was increased in increments of $10 \%$ of the average compressive strength in every cycle as shown in figure 2(b). The loaded and unloaded states in the loading program are referred to as $\mathrm{L}$ and $\mathrm{U}$ states, respectively. The load in the $\mathrm{L}$ states is given as a fraction of the 


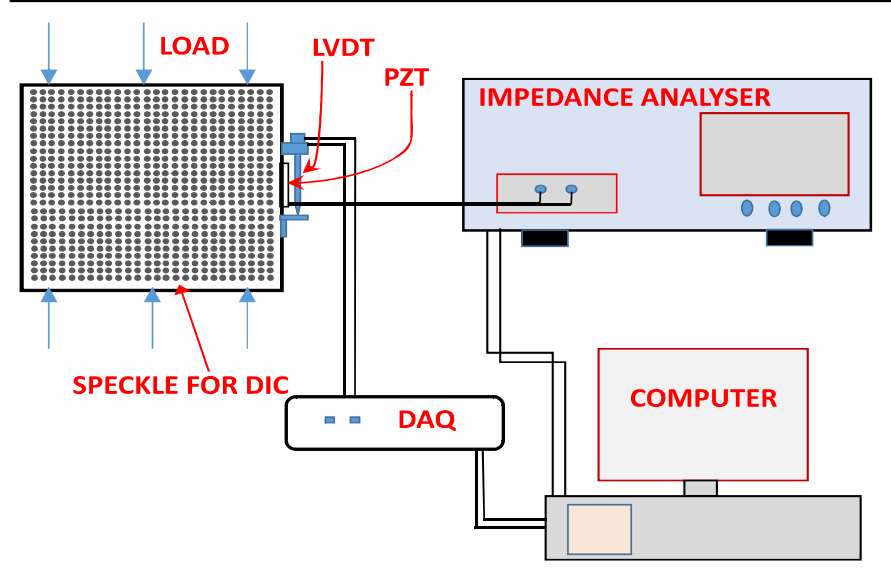

(a)

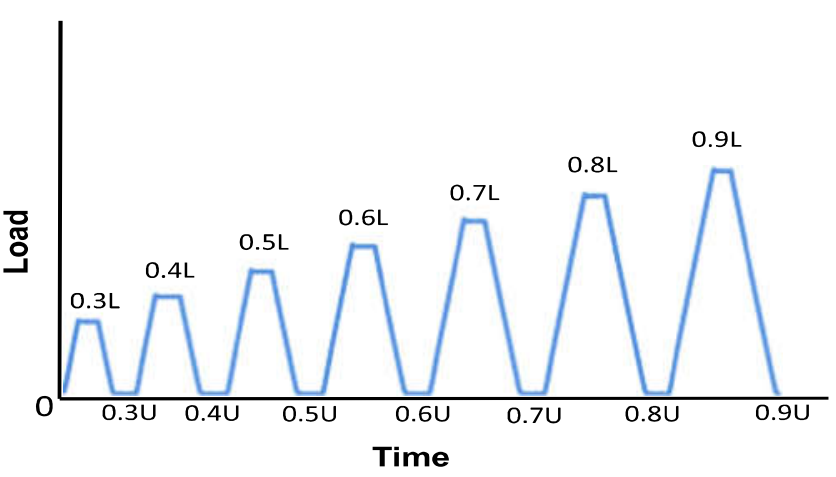

(b)

Figure 2. Compression testing of concrete: (a) test setup for EM impedance measurement; and (b) applied loading history.

average compressive strength (figure 2(b)). In the loading program, the load was cycled between a fixed lower load level and an increasing upper load level at a constant rate of $2 \mathrm{kN} \mathrm{s}^{-1}$. The load was held constant at the upper and lower load levels for a duration of $120 \mathrm{~s}$. EM impedance measurements were performed at the top and bottom of the loading cycle, when the applied load on the specimen was held constant. The specimens were loaded to failure from the $0.9 \mathrm{U}$ state. During the entire loading procedure, deformations were measured using two linear variable differential transformers (LVDTs) over a gauge length of $55 \mathrm{~mm}$. The LVDTs were mounted on opposite faces of the cubes and the average of the two LVDTs was used to measure the deformation of concrete.

The surface displacements on the surface of the concrete cube during the loading procedure were determined using a full-field optical technique based on digital image analysis called DIC. In preparation for the use of DIC technique, a sprayed- on speckle pattern was created on the surface of the specimen. Digital images of the specimen were captured using a camera of 5 mega-pixel resolution which was fitted with a $50 \mathrm{~mm}$ lens. The camera was placed at a distance of $1 \mathrm{~m}$ from the specimen surface. Uniform light intensity was ensured across the surface of the cube using normal white light.

The speckle pattern represents a random pattern, which gives a unique distribution of pixel gray level values. Crosscorrelation of the pixel gray levels between the image of the deformed specimen and the image of the specimen in the reference configuration is used to determine the two-dimensional displacement field on the surface of the specimen. Correlations are performed over small subsets of size equal to $32 \times 32$ pixels. The cross correlation analysis of the digital images was performed using the VIC-2D ${ }^{\mathrm{TM}}$ software (VIC2D ${ }^{\text {TM }}$ Reference Manual 2009). Surface displacements at each loading stage were calculated at each subset center, by evaluating the shape functions and their partial derivatives at the subset center. Strains were computed from the gradients of the displacements. For the setup used in this study, the random error in the measured displacement is in the range of 0.002 pixels. A conservative estimate of the resolution in strain obtained from the digital correlation was $5 \mu \varepsilon$ (Bruck et al 1989, Schreier and Sutton 2002).

Prior to initiating the loading program, load was cycled between $25 \%$ and $5 \%$ of the average compressive strength. The loading was initiated from a seating load $10 \mathrm{kN}$ (approximately $1 \%$ of the average compressive strength). For use of image analysis, a reference image corresponding to the unloaded state for computing strains was taken while the specimen was under seating load, before starting the loading program. Baseline electrical admittance signatures were also recorded from the PZT prior to starting the loading program.

\section{Experimental results}

Typical cyclic stress-strain responses obtained from NSC and HSC cubes using deformation measured by surface mounted LVDTs are shown in figure 3(a). The progressive decrease in mechanical stiffness and continued accumulation of plastic strain are evident with each additional cycle of loading. The secant compliance calculated between the top and bottom of each unloading cycle is shown in figure 3(b). It can be seen that there is an incremental decrease in stiffness with increasing load amplitude. In HSC, for load amplitude exceeding $0.4 \mathrm{~L}$ ( $40 \%$ of the average compressive strength), there is an almost linear decrease in stiffness with incremental load. There is an exponential decrease in stiffness with increasing load in NSC where the rate of change is increasingly higher for each additional load level. The plastic strains and hysteresis loops in HSC are significantly smaller indicating a significantly lower level of energy dissipation associated with damage when compared with NSC.

Contours of horizontal strain $\left(\varepsilon_{x x}\right)$ obtained using DIC for both NSC and HSC cubes in the $0.4 \mathrm{~L}, 0.7 \mathrm{~L}$ and $0.9 \mathrm{~L}$ states, are shown in figure 4 . The formation of cracks are identified with the localization of strain. From the strain contours, in NSC, the localization of the strain into major cracks is evident at $70 \%$ of the compressive strength. At the corresponding load level ( $0.7 \mathrm{~L}$ state), a significant lower level of damage associated with microcracks and small cracks are seen in 


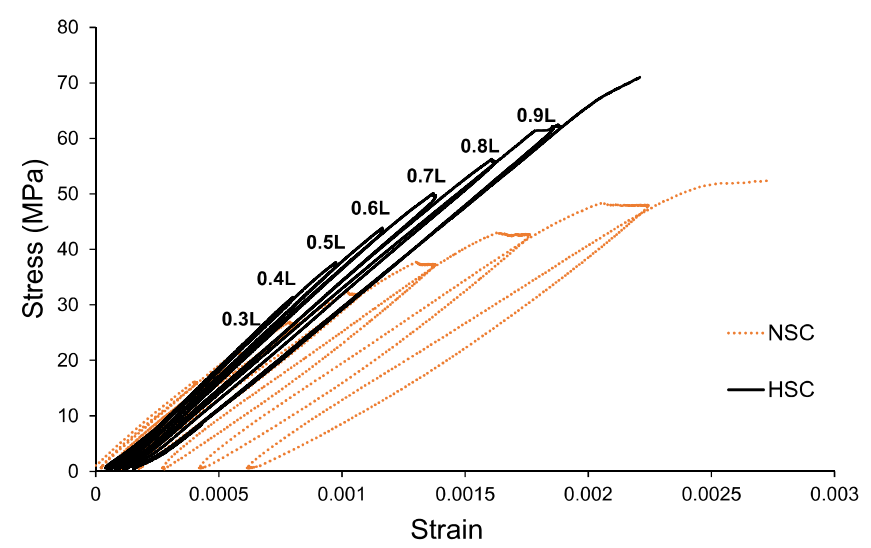

(a)

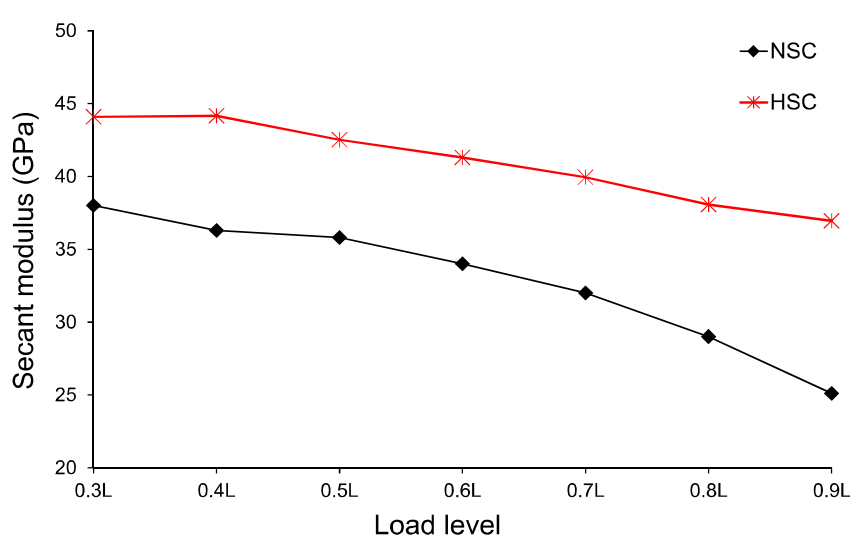

(b)

Figure 3. (a) responses of concrete cubes; and (b) secant stiffness as a function of load level.

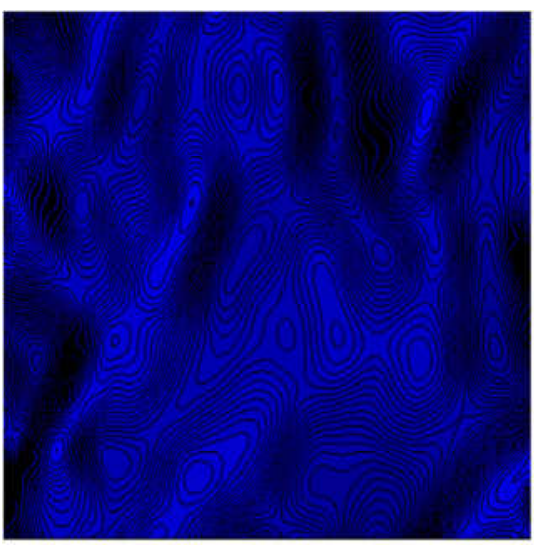

(a)

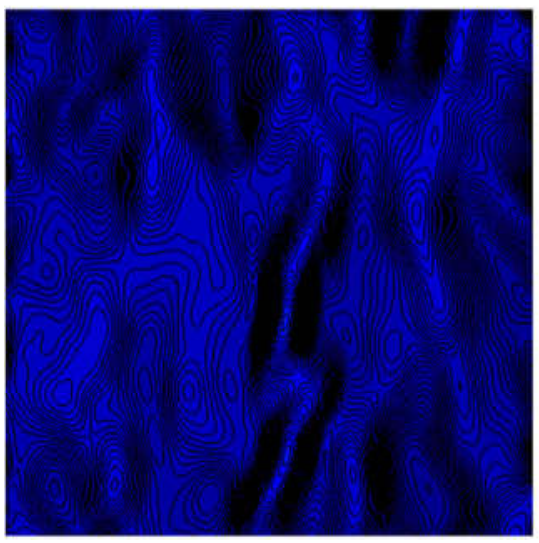

(d)

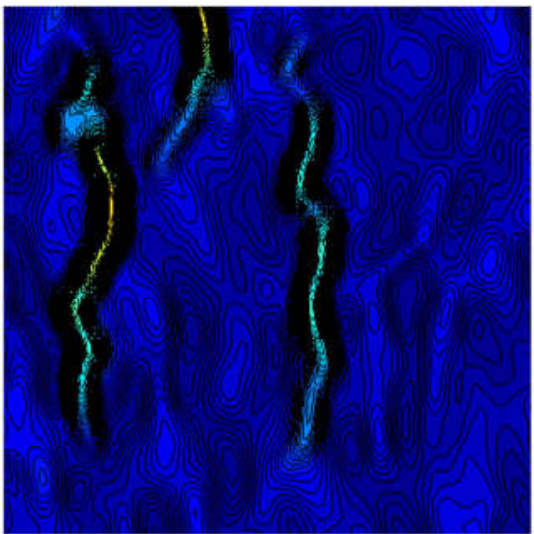

(b)

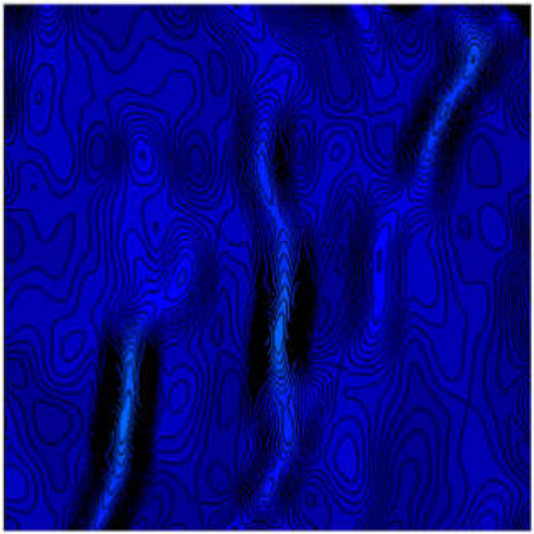

(e)

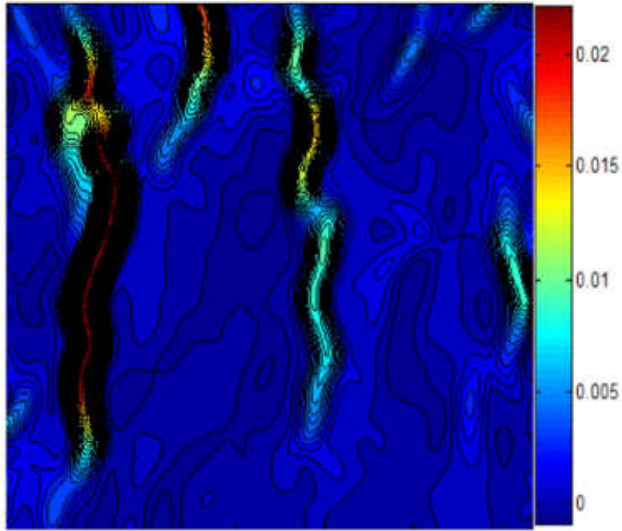

(c)

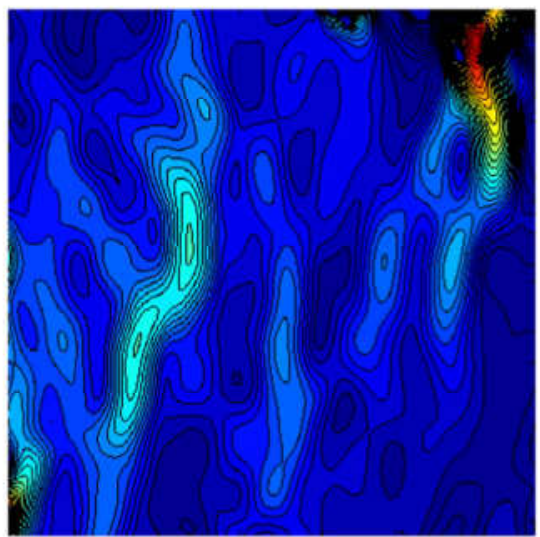

(f)

Figure 4. Strain contour $\left(\varepsilon_{x x}\right)$ at different load levels obtained from DIC in different loaded states in NSC at: (a) $0.4 \mathrm{~L}$; (b) $0.7 \mathrm{~L}$; (c) $0.9 \mathrm{~L}$; in $\mathrm{HSC}$ at (d) $0.4 \mathrm{~L}$; (e) $0.7 \mathrm{~L}$; (f) $0.9 \mathrm{~L}$.

HSC. At $0.7 \mathrm{~L}$, visible cracking could not be identified in HSC. At $0.9 \mathrm{~L}$, there is significant cracking associated with large strains in NSC. A major crack is seen in HSC at $0.9 \mathrm{~L}$. Therefore, NSC exhibits significantly higher level of distributed microcracks at lower loads which coalesces to form visible cracks at $0.7 \mathrm{~L}$ load level. In HSC, the overall level of damage was lower and the failure was brittle with lower accumulation of plastic strains. Visible cracks do not appear till $0.9 \mathrm{~L}$ load level.

The average $\varepsilon_{y y}$ (vertical strain) at the bottom and top of each load cycle obtained from DIC is shown in figure 5. The average $\varepsilon_{y y}$ was obtained by averaging $\varepsilon_{y y}$ from the DIC measurement across the front surface of the cube. The average strain at the top of a load cycle corresponds to strain due to 


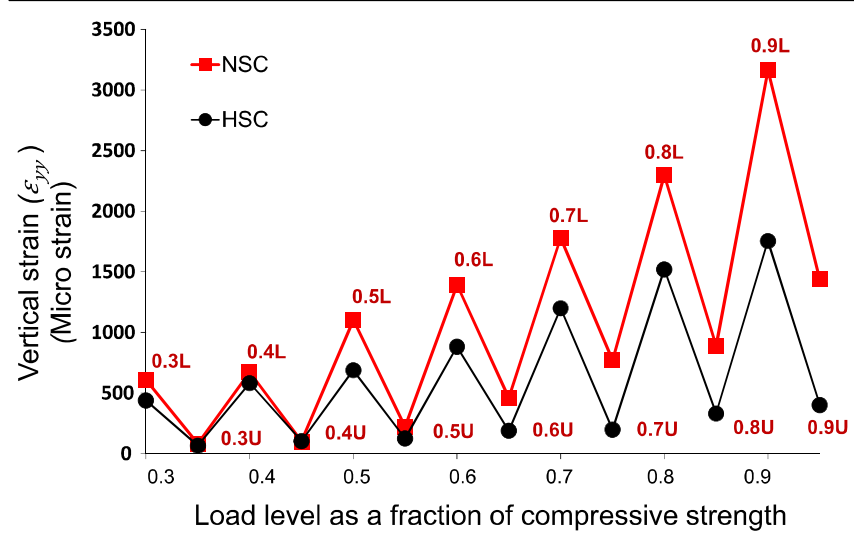

Figure 5. Average vertical strain $\left(\varepsilon_{y y}\right)$ at different $\mathrm{L}$ and $\mathrm{U}$ states obtained From DIC.

load-induced stress and damage. The average strain at the bottom of the load cycle is indicative of plastic strain due to damage. There is no measurable plastic strain in both NSC and $\mathrm{HSC}$ up to $0.4 \mathrm{~L}$ of the peak load (in the $0.4 \mathrm{U}$ state). As the loading increases, there is an increase in overall strain at the top of a load cycle and a steady accumulation of plastic strain upon unloading. The strain measures indicate a relatively larger plastic strain at corresponding load level (fraction of compressive strength) obtained from DIC measurements than from the surface mounted gages. This is due to the inclusion of localized strains from cracking over the entire surface, which are accurately captured in the DIC measurements. After localization of damage, the surface gages may not detect the presence of the crack unless located over it, while it is reflected in the averaged surface measurement of DIC. The measurements indicate that NSC has a higher level of damage in the material when compared with HSC at the corresponding load level. In the $0.9 \mathrm{U}$ state, there is significant strain, on the order of $1000 \mu \varepsilon$ in the NSC. The strain in HSC in the $0.9 \mathrm{U}$ state is significantly smaller. Findings from DIC measurements suggest that the strain in the substrate is influenced by both the level of damage and the applied stress. Under applied loading, above $40 \%$ of the compressive strength, an increment in load produces an increment in strain associated with both effects.

The conductance signatures associated with the second resonant peak in the EM admittance response of PZT bonded to concrete cubes, for both NSC and HSC, at different levels of applied load are shown in figure 6. The conductance peak is associated with the second resonant peak is centered on 253 and $255 \mathrm{kHz}$ for NSC and HSC, respectively. Conductance spectra exhibit an increase in peak amplitude at successively higher load levels. There is also an overall increase in the amplitude at all frequencies. At corresponding load levels (fraction of compressive strength), larger shifts are observed in NSC when compared with HSC.

Changes in the second resonant peak in the conductance spectrum of the PZT attached to concrete block after unloading from different load levels are shown in figures 7(a) and (b) for NSC and HSC, respectively. It can be seen that on unloading from successively higher load levels, consistently there is a leftward shift in the spectrum resulting from a larger relative decrease in amplitude at frequencies higher than the centerline frequency in the spectrum. The magnitude of shift and decrease in amplitude of the spectrum are consistent with the level of damage indicated by the change in compliance and increase in substrate plastic strain obtained from mechanical measurements. For unloading from a given load level, the higher level of damage observed in NSC coincides with the larger shift in the spectrum and a larger decrease in amplitude of conductance. Visible cracking in NSC, when the load level was $0.7 \mathrm{~L}$, is associated with a larger shift in frequency to the left accompanied by a broadening of the peak. A slight flattening of the peak is also observed when the visible crack appeared. In NSC, there is an abrupt change in the shape of the spectrum at $0.9 \mathrm{~L}$ of the peak load, which produces significant peak broadening coinciding with significant localized damage in the form of visible cracking in the vicinity of the PZT. Visible cracking in HSC at $0.9 \mathrm{~L}$ also produces significant decrease in amplitude and shift in the peak.

The spectrum of susceptance obtained from the measured electrical admittance of the bonded PZT in the $\mathrm{U}$ and $\mathrm{L}$ states are shown in figures 8(a) and (b), respectively. The low frequency susceptance signature, which is related with the structural modes (or response) is also shown in the figure. The overall change in the slope of the low-frequency susceptance signature had previously been shown to be sensitive to stress under increasing loading recorded from PZTs attached to thin aluminium specimens (Annamdas et al 2007). The slope of the low-frequency susceptance signature does not exhibit any significant or consistent variation with loading. Unlike the case of aluminium specimen, increasing stress level in concrete specimen is associated with an increase in the level of damage. The combined effect of increasing stress and damage in concrete may likely result in no change in the slope of the susceptance spectrum.

The response of the PZT to an imposed strain was evaluated by applying small strains directly on the PZT. The PZT was subjected to an imposed strain using a digital micrometer. A photograph of the test setup is shown in figure 9(a). The direction of applied strain in the PZT was kept the same as in the concrete specimen. The conductance signature of the third resonant mode of the free PZT (corresponds to the second peak in the conductance spectrum of bonded PZT) for different levels of imposed strain is shown in figure 9(b). The changes in peak of the conductance spectrum produced by imposed strains results in a shift in the central frequency to a higher value and an increase in the amplitude of the peak. The observed behavior of the PZT confirms that an applied compressive strain influences the impedance response of a PZT resulting in a change in the resonant behavior of the PZT. For a PZT bonded to a concrete substrate, the incremental strain associated with an increment of load is expected to introduce increases in the frequency and the amplitude of the resonant peak in the conductance response. 


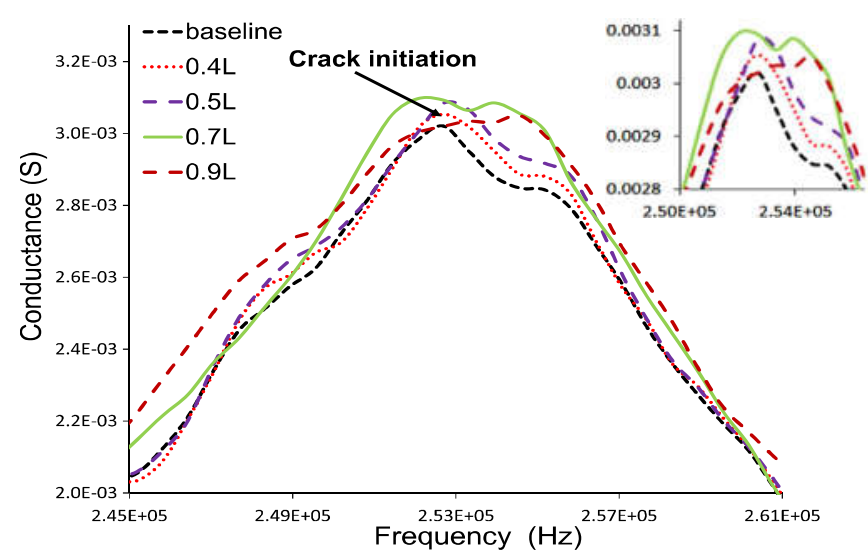

(a)

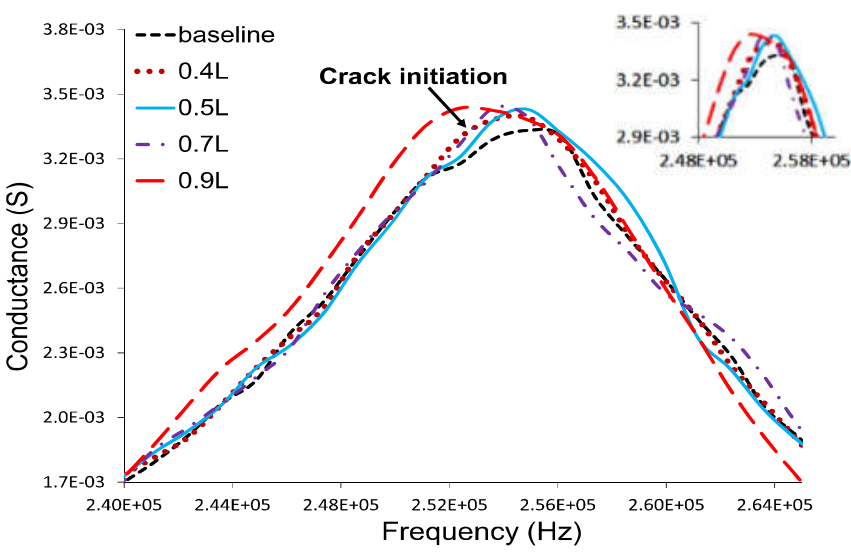

(b)

Figure 6. Conductance spectra close to second peak in the EM admittance response of PZT bonded to concrete cube at different levels of load in: (a) NSC; and (b) HSC.

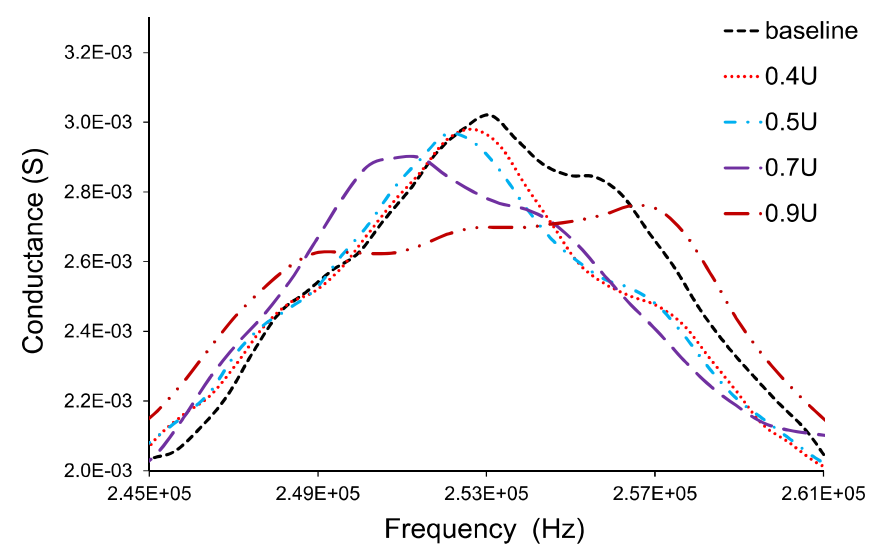

(a)

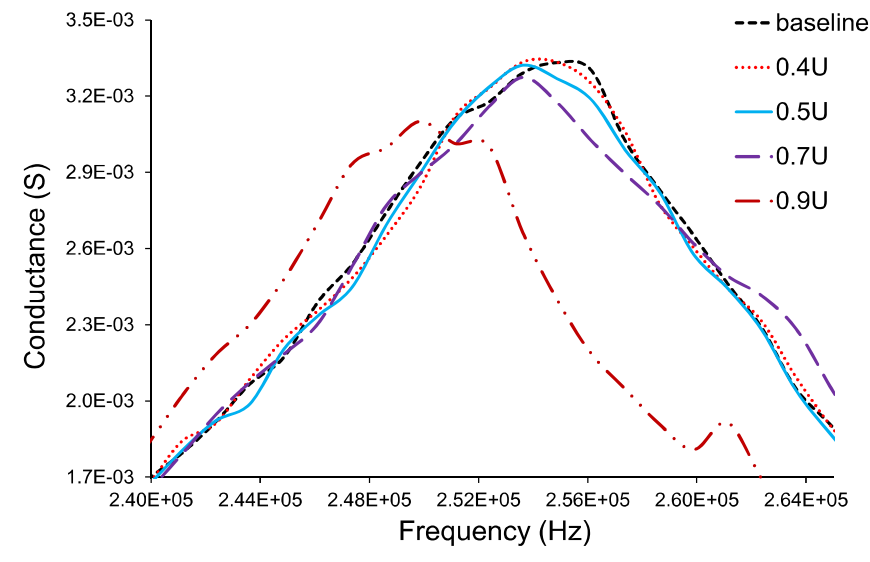

(b)

Figure 7. Conductance spectra close to second peak at different levels of damage in: (a) NSC; and (b) HSC.

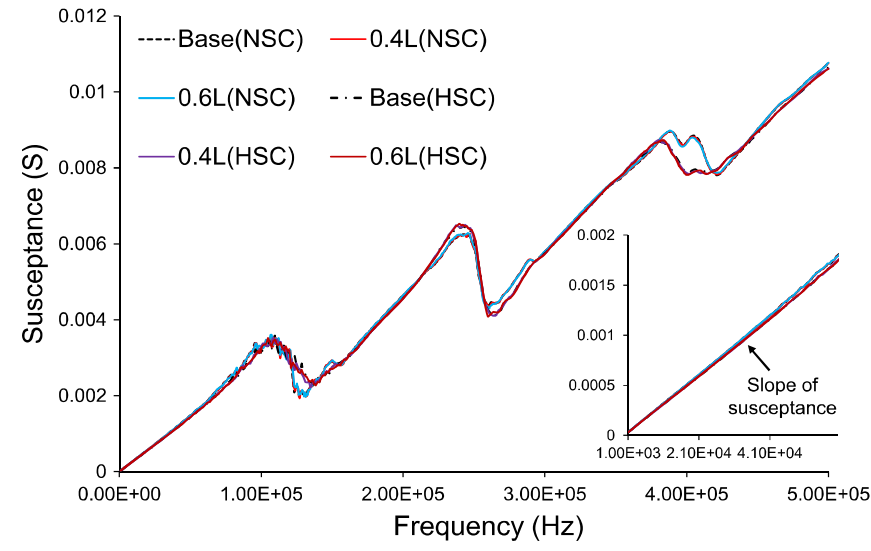

(a)

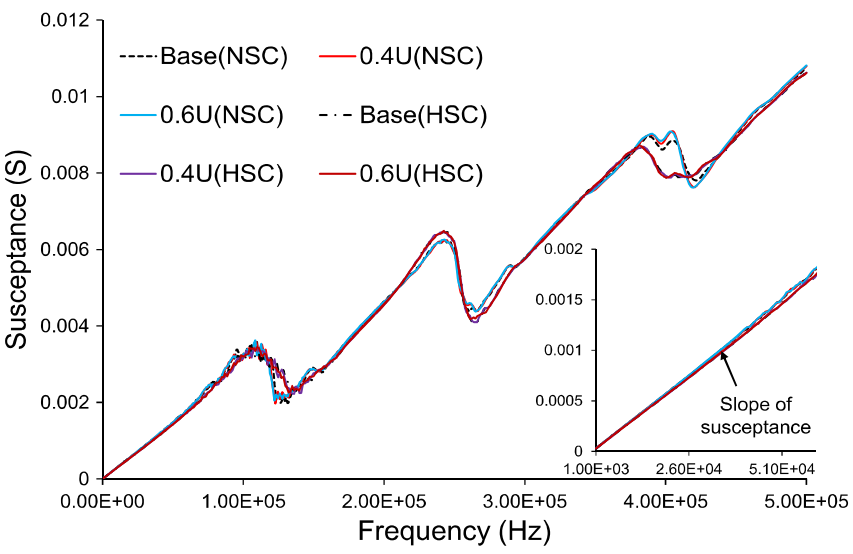

(b)

Figure 8. Susceptance signatures obtained from the EM impedance measurement of bonded PZT in: (a) L states; and (b) U states.

\section{Analysis of the results}

The results of experimental program clearly indicate that the change in the measured conductance in the EM admittance response of a PZT attached to a concrete substrate depends upon the level and type of damage and the stress in the substrate material. The observed changes in the PZT resonant peaks in the conductance spectrum can be interpreted considering the level of damage, stress in substrate material and strain in the PZT as shown schematically in figure 10. The 


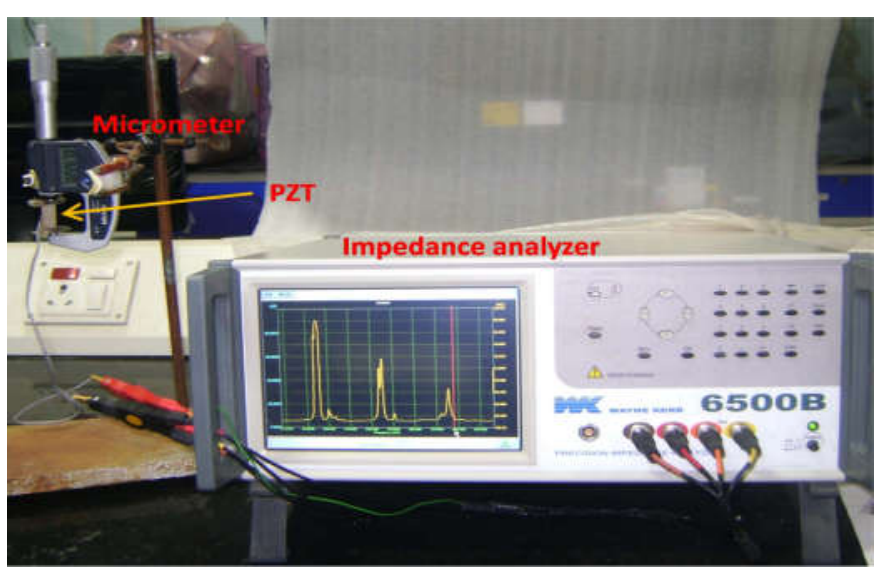

(a)

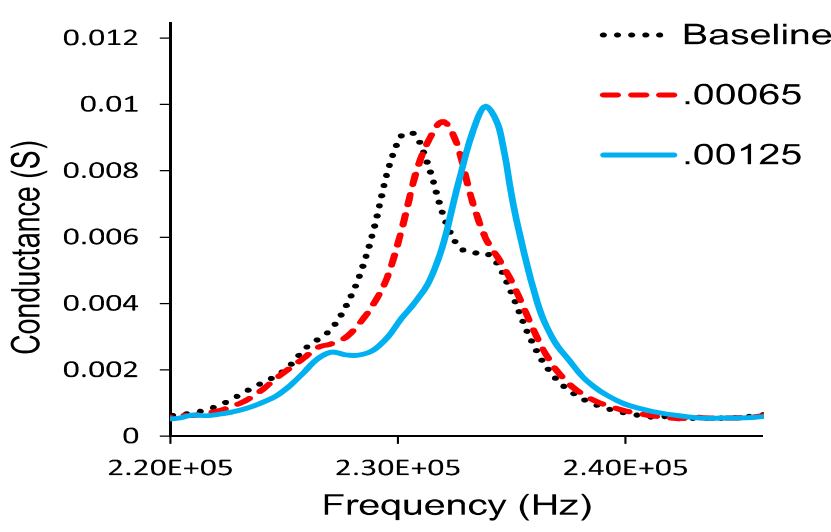

(b)

Figure 9. (a) Micrometer setup for imposed strain measurement; (b) conductance spectra close to second peak of the PZT at different imposed strain levels.

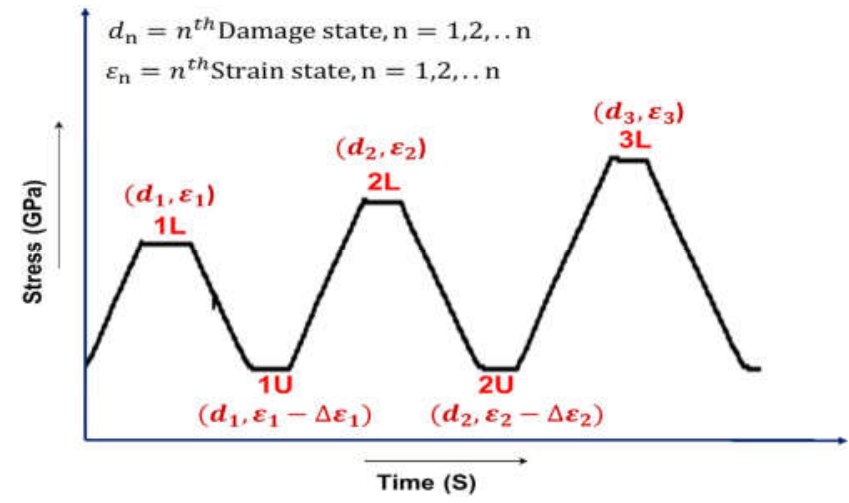

Figure 10. Schematic representation of strain and damage levels in substrate.

increment in strain levels between two adjacent $\mathrm{U}$ levels is associated with an increment in the plastic strain. Between two adjacent $U$ states, for a given change in the substrate plastic strain, the strain induced in the PZT depends on the material compliance of concrete. The EM impedance response of the bonded PZT depends on the mechanical impedance of the substrate (and hence material compliance), which directly depends on the level of damage. Therefore, comparison of adjacent $U$ states provides information about incremental levels of damage, in the absence of stress in the substrate. A measure of the mechanical impedance of the substrate is obtained from the mechanical stiffness of the specimen. As the level of damage increases, there is an increase in the compliance of the material (and hence a decrease in its mechanical impedance). Consistently there is a downward shift in the resonant frequency along with an overall leftward shift in the spectrum recorded in the U states (shown in figure 7). The increase in the level of damage in the form of microcracks is also associated with an increase in the level of damping, which produces a decrease in frequency of the peak (Narayanan and Subramaniam 2016). While the increase in material compliance produces an increase in amplitude, increase in damping results in a reduction in the amplitude of the peak. The amplitude variation in the resonant peak in the conductance spectrum depends on the relative influence of damage on the elastic modulus and the damping of the material (Narayanan and Subramaniam 2016).

On unloading the specimen from the $\mathrm{L}$ state to the $\mathrm{U}$ state, the stress in the substrate is reduced, while the level of damage is the same. In the L state, there is an elastic strain in the substrate associated with applied stress, in addition to the permanent strain produced by damage in the substrate. In the loaded state, the elastic strain in the substrate depends on the material compliance, which in turn depends on the level of damage. Since the level of damage is the same, comparing the $\mathrm{L}$ state with the immediate $\mathrm{U}$ state allows for assessing the influence of stress in the substrate (and hence of imposed strain in the PZT) on the impedance response of a PZT for a given level of damage. The difference in the substrate strains in these two states is attributed to elastic strain, which is directly proportional to the stress. A comparison of the conductance spectra from specimens at the top of the load cycle and after unloading from the load envelope is shown in figure 11 . It can be seen that compared to the $\mathrm{U}$ state the corresponding L state exhibits an increase in the amplitude and a rightward shift in the spectrum. The results considering the increment in the PZT strain produced by applied stress in the substrate are consistent with the findings from direct application of strain on the PZT (shown in figure \&(b)). At the very high load level, corresponding to $0.9 \mathrm{~L}$, noticeable decreases in frequency and amplitude are observed in the unloaded state for both NSC and HSC. The localized cracking in the vicinity of the PZT in NSC also produces a significant change in shape associated with broadening of the resonant peak. However, the level of damage is not that evident considering the response under load. The results indicate that the increment in the level of substrate stress has a dominant effect on the conductance of a bonded PZT.

Strain induced in the PZT due to increasing stress in the substrate increases the amplitude of the conductance and produces a frequency shift to higher values. An increase in material compliance due to damage produces a leftward shift 


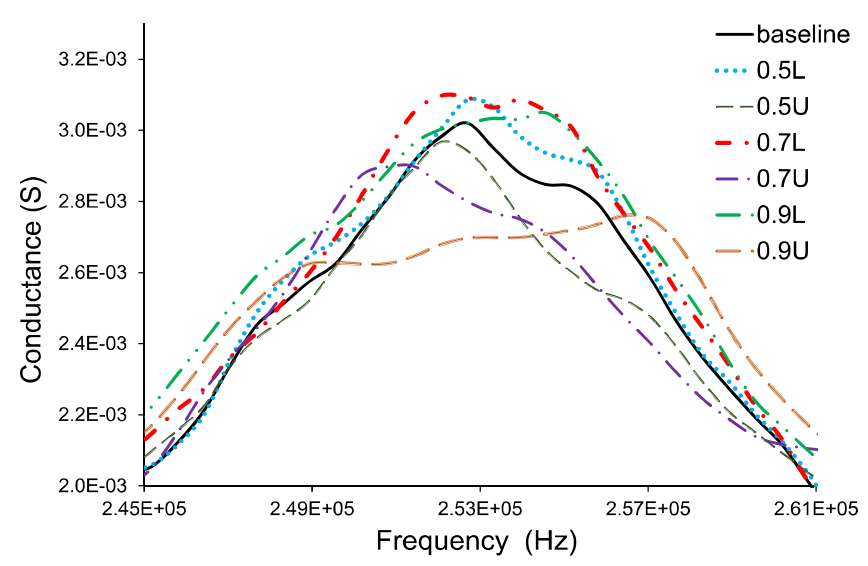

(a)

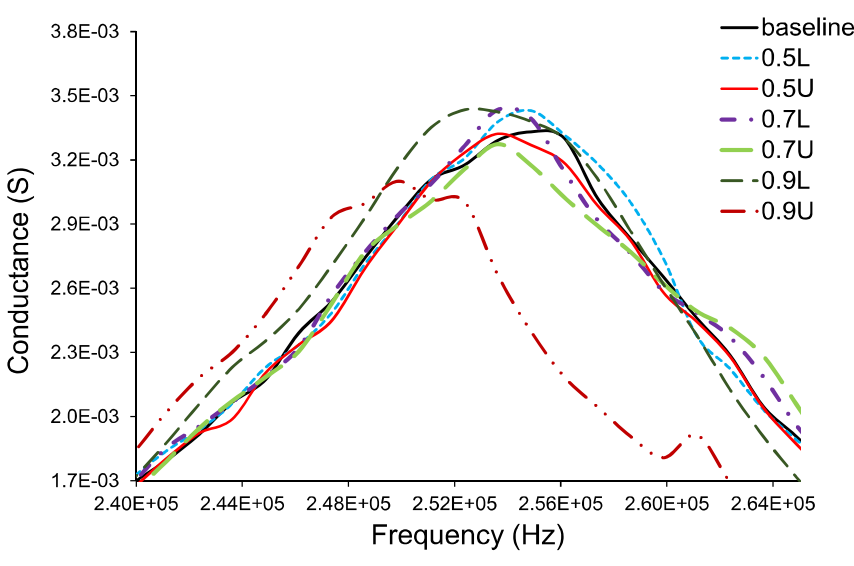

(b)

Figure 11. Conductance spectra close to second peak in the EM admittance response of bonded PZT from the L and U states: (a) in NSC; and (b) in HSC.

in frequencies, with a change in amplitude which depends on the relative influence of damping and increasing material compliance. The frequency shift in the EM spectrum is influenced by the counteracting effects produced by imposed strain and change in material compliance.

\subsection{Measures of quantification}

A quantification of the observed frequency shifts and changes in the shape of the spectrum induced by applied stress and damage is now attempted. Measures of change include the shift in frequency, the change in bandwidth of the peak and the root mean square deviation (RMSD). A measure of the shift in frequency is obtained using a measure of centroidal frequency of the resonant peak in the conductance spectrum given as

$$
\bar{f}=\frac{\int_{f_{1}}^{f_{2}} f Y \mathrm{~d} f}{\int_{f_{1}}^{f_{2}} Y \mathrm{~d} f},
$$

where $Y$ is the conductance at a given frequency, $f_{1}$ and $f_{2}$ are fixed frequencies. Shift in frequency, $\Delta f$ is calculated using equation (5), where $\bar{f}_{c}$ is the centroidal frequency of the spectrum and $\bar{f}_{0}$ is the initial centroidal frequency

$$
\Delta f=\left|\bar{f}_{c}-\bar{f}_{0}\right| \text {. }
$$

The broadening effect of the peak at each damage level was quantified using bandwidth of the peak (BW). The bandwidth represents the distance between the two points in the frequency spectrum where the signal is $\frac{1}{\sqrt{2}}$ of the maximum amplitude (half power). The bandwidth is defined with respect to the full width at $\frac{Y}{\sqrt{2}}$ as shown in figure 12 . Percentage change in bandwidth is calculated using equation (6)

$$
\Delta \mathrm{BW}(\%)=\frac{\left(\mathrm{BW}_{c}-\mathrm{BW}_{0}\right)^{*} 100}{\mathrm{BW}_{0}} .
$$

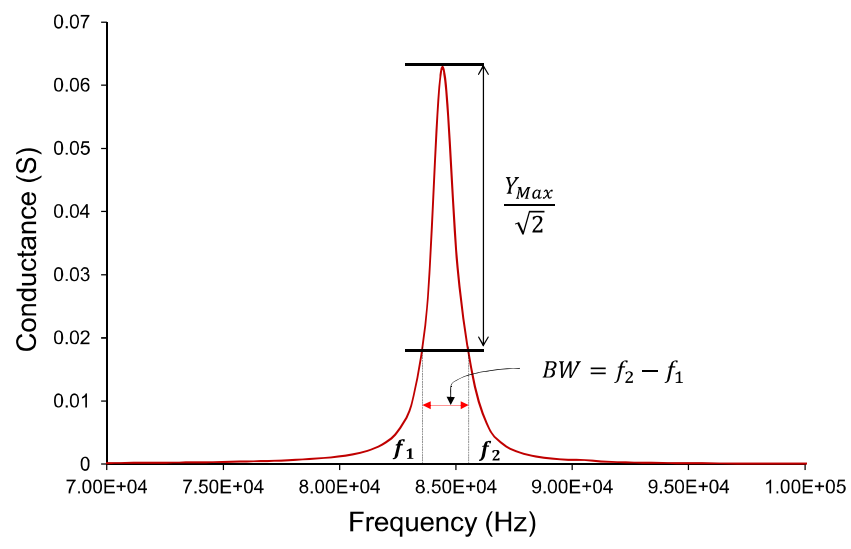

Figure 12. Bandwidth of resonant peak.

The RMSD is used to calculate the difference between values of baseline measurement of conductance signature and the signatures in the different $\mathrm{L}$ and $\mathrm{U}$ states. The RMSD for second resonant peak with respect to the baseline measurement were calculated in the frequency range $(245-260 \mathrm{kHz})$ using equation (7), where. $x_{i}$ and $y_{i}$ are the baseline and values of conductance at $\mathrm{L}$ or $\mathrm{U}$ states, respectively

$$
\operatorname{RMSD}=\sqrt{\frac{\sum_{i=1}^{N}\left(y_{i}-x_{i}\right)^{2}}{\sum_{i=1}^{N} x_{i}{ }^{2}}} .
$$

Figure 13 shows the frequency shift of the conductance peak in the $\mathrm{L}$ and the $\mathrm{U}$ states. The frequency shift for the $\mathrm{L}$ and $U$ states have been calculated with reference to the centroidal frequency recorded before initiating loading. While shifts are noticeable in the $U$ states, there is no change in centroidal frequency in the L states. For both NSC and HSC there is a continuous frequency shift after $0.4 \mathrm{U}$. There is a consistent increase in the centroidal frequency, with increasing damage level in the successive $U$ states. The frequency shift in the $U$ states follows a trend which is similar to the observed decrease in stiffness obtained from mechanical measurements; there is an exponential shift at higher load 


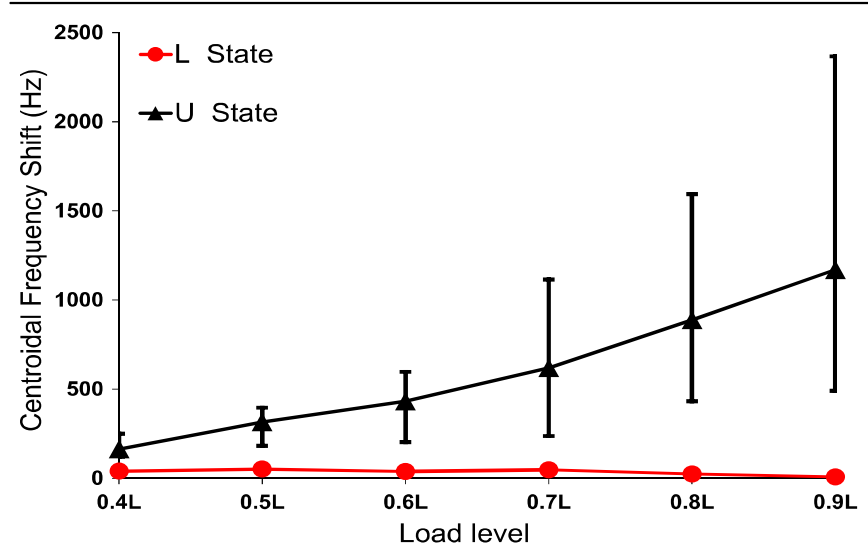

(a)

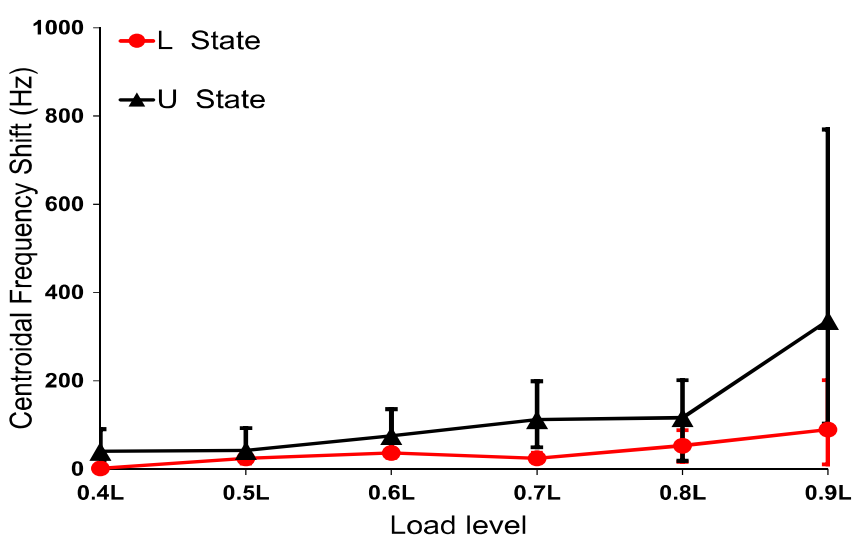

(b)

Figure 13. Frequency shift in resonant mode with unloading in the U states: (a) in NSC; and (b) in HSC.

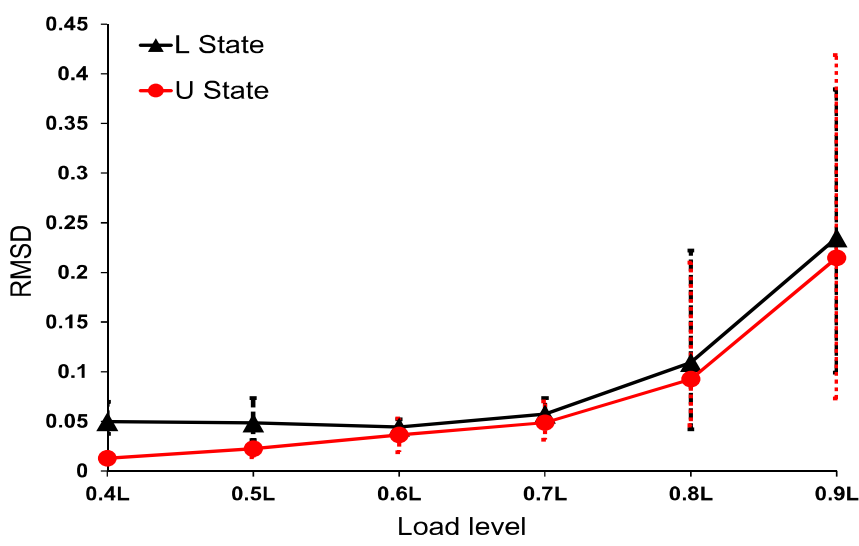

(a)

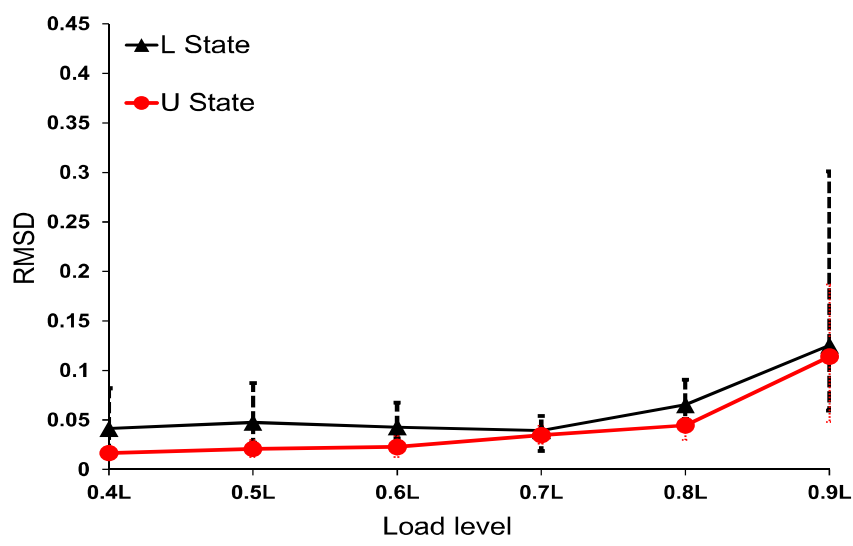

(b)

Figure 14. RMSD of resonance peak in the L and U states: (a) in NSC; and (b) in HSC.

ratios in NSC, while the HSC exhibits a linear increase. At higher level of damage the peak was flattened and there was no distinctive peak. The centroidal frequency therefore provides a good indication of damage level in the $U$ state. The insensitivity of resonance peak frequency shift in the $\mathrm{L}$ states is due to the combined influence of damage in the substrate and imposed strain on the PZT, which produce counteracting effects.

Change in the RMSD values for the $\mathrm{L}$ and $\mathrm{U}$ states in NSC and HSC are shown in figure 14. The RMSD values obtained in both the $\mathrm{L}$ and $\mathrm{U}$ states are generally in agreement for loads higher than after $70 \%$ of the compressive strength. While the RMSD values obtained from the L states are essentially constant up to $0.7 \mathrm{~L}$. The magnitude of change in the U states at any load level is higher in NSC than the corresponding change observed in HSC. The RMSD values in the U states have previously been shown to correlate well with the damage level (Narayanan and Subramaniam 2016). The RMSD value obtained in the $\mathrm{L}$ state is reflective of both damage and imposed strain. The observed trend in the RMSD indicates that the influence of imposed strain and damage produce counteracting effects. The influence of damage is evident after $0.7 \mathrm{~L}$. Thus it can be concluded in the presence of imposed strain, RMSD shows a change only at very high levels of damage in the material. At low stress levels, the counteracting influences of damage and stress produce little or no change in the RMSD.

The changes in bandwidth of the conductance spectrum in the $\mathrm{U}$ and $\mathrm{L}$ states for NSC cube are shown in figure 15 . No change was obtained in the bandwidth for HSC cubes up to a very high load levels. This suggests that the change in bandwidth is more related to localized damage as observed in NSC specimen. In NSC cubes, the bandwidth measured in the $\mathrm{U}$ states indicates a continuous increase with an increase in damage level. In the $\mathrm{L}$ states, the increase in bandwidth is minimal at low stress levels and an increasing trend can be observed under higher load ratios. Thus the strain dominant and damage dominant effect on conductance signature can be differentiated from the change in bandwidth.

\subsection{Identification of stress induced damage}

A measure of normalized conductance given in equation (8) is introduced to identify the amplitude shifts from the baseline 


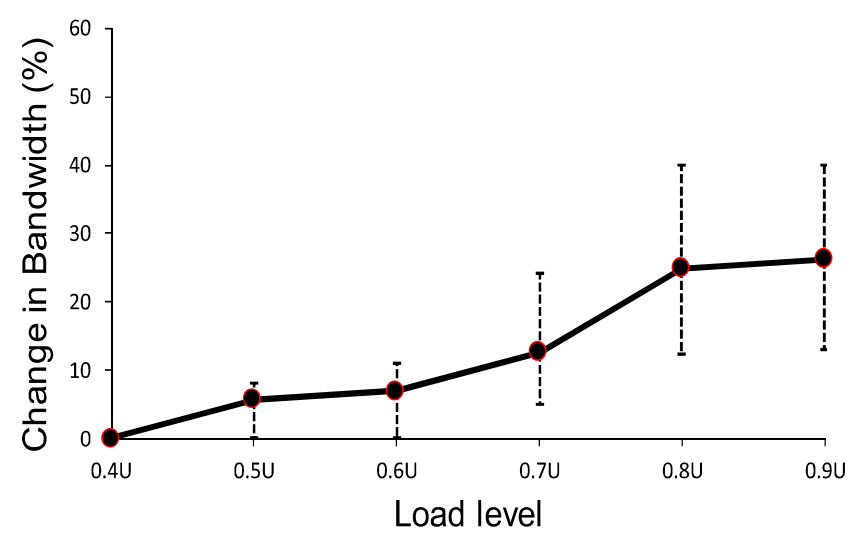

(a)

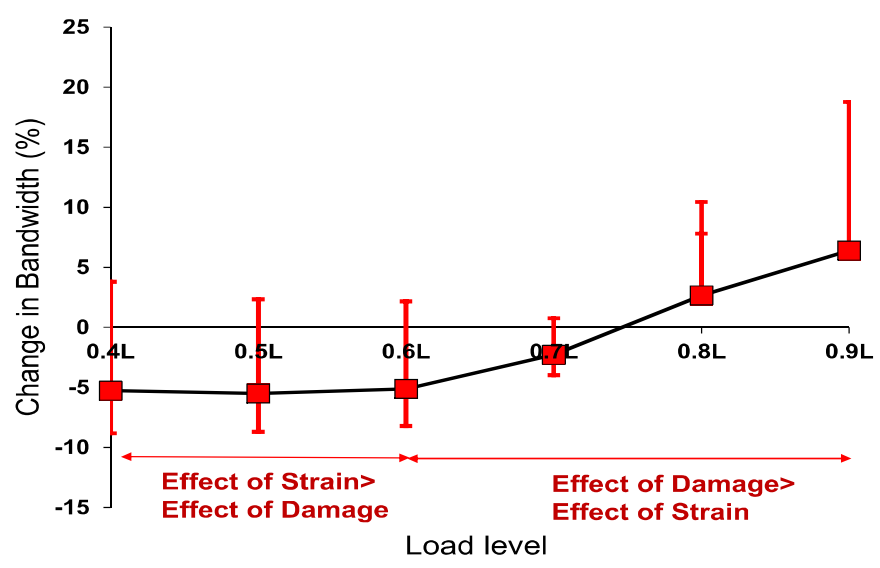

(b)

Figure 15. Percentage change in bandwidth in: (a) U states; and (b) L states.

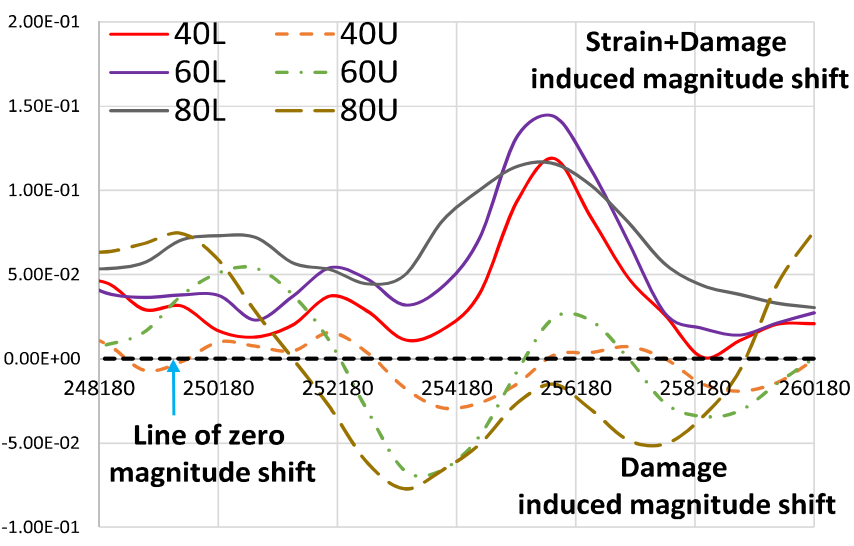

(a)

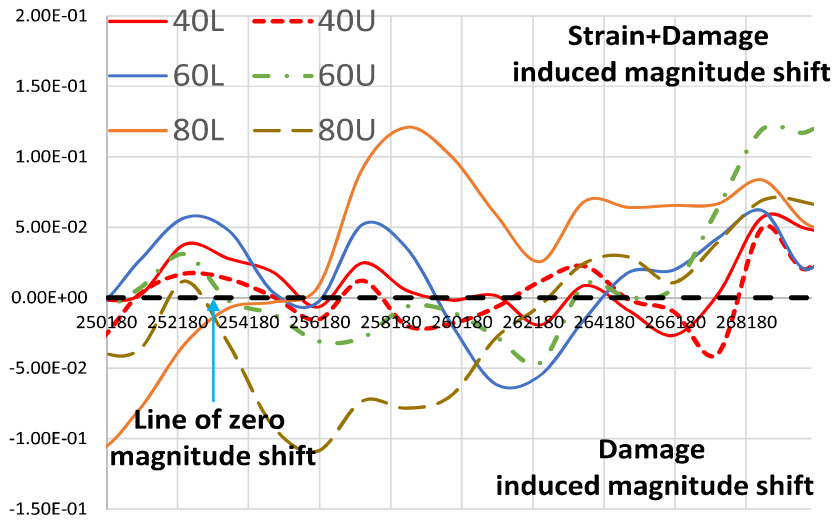

(b)

Figure 16. Conductance (normalized) signature in the different $\mathrm{L}$ and $\mathrm{U}$ states: (a) NSC; and (b) HSC.

signature produced by damage and induced strain.

$$
\text { Normalized signature }\left(Y_{N}\right)=\frac{Y_{i}-Y_{B}}{Y_{B}},
$$

where $Y_{i}$ is the conductance at a given frequency in the $\mathrm{L}$ or $\mathrm{U}$ states and $Y_{B}$ is the corresponding baseline conductance. Figure 16 shows the normalized conductance signature at different load levels for NSC and HSC. The strain induced conductance changes can be clearly identified from the normalized conductance values. An upward shift can be seen qualitatively in the normalized signature from the L states. Consistent shifts are produced with increasing stress level in the substrate, irrespective of damage level. The magnitude shift of normalized signature in the L states for HSC cube is lower even than the shifts observed in the NSC cubes at the corresponding load levels. This can be correlated with the overall lower strain in the L states in HSC as seen in figure 5.

To quantify the observed shifts in the normalized conductance signature, the centroid of the normalized conductance, $\bar{Y}$ was computed as shown in equation (9)

$$
\bar{Y}=\frac{\int_{f_{1}}^{f_{2}} Y_{N}^{2} \mathrm{~d} f}{2 \int_{f_{1}}^{f_{2}} Y_{N} \mathrm{~d} f},
$$

where $f_{1}$ and $f_{2}$ are fixed frequencies

The trends in the values of $\bar{Y}$ in the $\mathrm{L}$ and $\mathrm{U}$ configurations are shown in figures 17(a) and (b) for NSC and HSC, respectively. The influence of strain in the substrate can be clearly identified with a positive shift (an upward movement) of the centroid. The magnitude of the shift in the L states corresponds with the level of stress in the substrate; a larger shift is observed in NSC when compared with HSC. With an increase in the substrate stress, up to $0.7 \mathrm{~L}$ in NSC and $0.9 \mathrm{~L}$ in HSC, there is an upward shift in $\bar{Y}$. The values of $\bar{Y}$, upon unloading to the $\mathrm{U}$ states show no change. The difference between the $\mathrm{L}$ and the immediate $\mathrm{U}$ state is the imposed strain in the PZT due to stress in the substrate. Comparing two U states, there is an increase in the level of damage, which produces additional plastic strain and an increase in material compliance. The counteracting influences of increase in material compliance and increasing plastic strain (and hence 


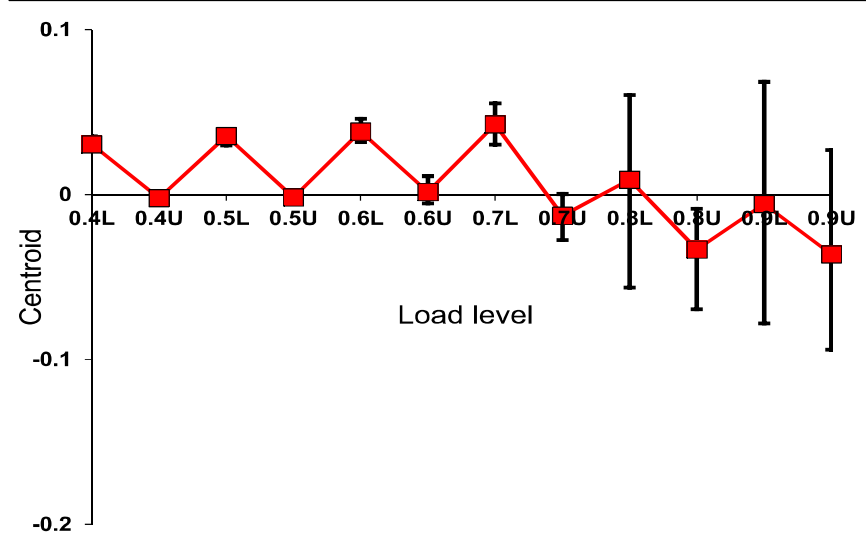

(a)

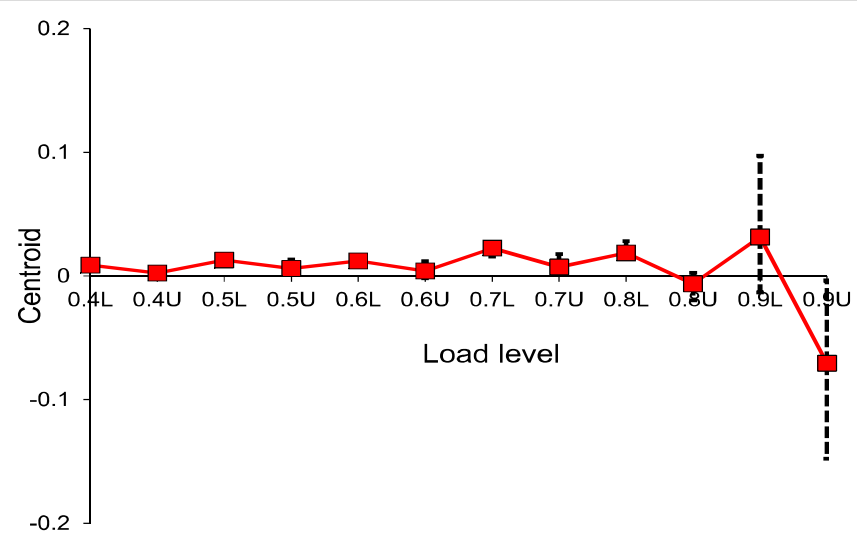

(b)

Figure 17. Position of centroid in the L and U states: (a) NSC; and (b) HSC.

imposed strain on PZT) result in no shift in the centroid of the normalized amplitude.

Localization of damage and the formation of a crack produces a downward shift in the $\bar{Y}$, which occur close to $0.7 \mathrm{~L}$ and $0.9 \mathrm{~L}$ in NSC and HSC, respectively. The crack formation produces an overall downward shift in the centroid in both the $\mathrm{L}$ and $\mathrm{U}$ states. The downward movement of centroid in the $\mathrm{L}$ states shows that the influence of damage is more dominant on resonant peak behavior after the localization into a visible crack.

The effect of stress and microcrack damage can be decoupled using the normalized conductance signature as a quantitative measure. The absolute value of centroid of the normalized spectrum associated with the second resonant peak of a PZT bonded to a concrete substrate curve gives the identification of stress and microcrack damage induced change. The downward shift of the centroid can therefore be taken as indicator of localized damage in the form of cracks. The upward shift of the centroid is indicative of increasing stress in the substrate.

\subsection{Identification of damage and strain}

The results of the quantitative analysis can be assimilated to provide an understanding of the influence of damage and stress. The influence of increasing damage in the material substrate can be identified with three effects: (a) increase in material compliance; (b) increase in plastic strain; and (c) increase in material damping. While increase in material compliance effects a leftward shift in spectrum, the increase in plastic strain (increases the imposed strain on PZT) produces a rightward shift. In the absence of stress in the substrate, such as in the U states, while there are counteracting influences of the two effects, the influence of decrease in compliance is dominant producing a net shift in frequencies (as seen in figure 13). Considering the three effects of damage while (a) and (b) produce an increase in the amplitudes, damping produces a decrease in the amplitude. In the absence of stress, the influence of these counteracting effects is to produce no change in the amplitude centroid of the spectrum (as seen in figure 17).

Stress in the substrate produces an additional imposed strain in the PZT, which produces an increase in the frequency and the amplitude. For a given level of damage, on increasing the stress in the substrate, there is a net increase in the amplitude of the spectrum and no shift in the centroidal frequency of the spectrum.

\section{Findings and summary}

The use of EM impedance-based monitoring of concrete structures is evaluated in this study. Damage in concrete has been evaluated using full-field displacements measurements on the surface of a concrete cube obtained from DIC. The evolution of micro- cracks, which coalesce leading to localization of damage in the form of cracks is shown to correlate with conductance obtained from EM impedance measurements from PZT bonded to the concrete substrate. The results of the experimental program presented here clearly show that the conductance spectrum obtained from the EM impedance measurement of a bonded PZT reflects changes in the local mechanical compliance of the substrate due to damage. Damage in the form of microcracks is found to influence the electrical conductance through a change in substrate compliance. Increasing damage is shown to produce a decrease in the magnitude and frequency of the resonant peak of the PZT in the electrical conductance spectrum of the bonded PZT.

Imposed strain is shown to influence the conductance signature from an EM impedance measurement of a PZT. Increments in imposed strain in a bonded PZT are associated with an increase in elastic strain in concrete produced by increments of stress and permanent strains associated with the level of damage in the material. Change in imposed strain on the PZT produces changes in the resonant peak which are counter acting to the influence of damage. In the presence of stress, the observed change in the resonant peak signature in the conductance spectrum do not show any significant changes for low levels of damage associated with microcracking. 
With an increase in stress, as the damage in the material increases in a nonlinear manner, the influence of damage is reflected in most measures of damage such as the frequency shift, RMSD and band width calculated from the resonant peak. At low levels of damage, associated with distributed microcracking, the normalized conductance peak signature accurately reflects the level of stress in the substrate.

The use of EM impedance measurements for evaluating damage in a concrete substrate requires careful consideration of the stress in the substrate in addition to the type and level of damage. An increment in the magnitude of stress is associated with an increment in the strain and also the level of damage. The presence of damage and the type of damage also influence the strain in the substrate for a given applied stress. Localization of damage in the form of cracks results in a nonlinear increase in strain in the substrate. The behavior of the bonded PZT is influenced by the substrate compliance as influenced by the amount and type of damage. The localization of damage in the form of cracks is reflected in the nonlinear decrease in the mechanical stiffness. Therefore the extent and type of damage influence both the strain in the substrate and the mechanical compliance of the substrate.

In a real structure, the possibility of unloading to establish the true level of damage without the influence of applied stress does not exist. Features of the resonant peak in the conductance spectrum of a bonded PZT are indicative of the state of substrate in terms of the level of strain and the type and extent of damage. At low levels of damage, the increment in the substrate stress can be identified using the shifts in the normalized conductance signature. In a loaded state, localization of damage and formation of cracks can be identified using the shifts in the bandwidth of the resonant peak and the downward shift in the centroid of the normalized conductance signature.

\section{Acknowledgments}

This work was supported by Grant No. SB/S3/CEE/0013/ 2013 from the SERB Unit of the Department of Science and Technology, Ministry of Science and Technology, India.

\section{Q3 References}

Annamdas V G M, Yang Y and Soh C K 2007 Influence of loading on the electromechanical admittance of piezoceramic transducers Smart Mater. Struct. 16 1888-97

Q4 ANSI/IEEE Std. 176-1987 IEEE Standard on Piezoelectricity

Bhalla S and Soh C K 2004a Structural health monitoring by piezoimpedance transducers: I. Modeling J. Aerosp. Eng. 17 154-65

Bhalla S and Soh C K 2004b Structural health monitoring by piezoimpedance transducers: II. Applicaions J. Aerosp. Eng. 17 166-75

Bruck H A, McNeill S R, Sutton M A and Peters W H 1989 Digital image correlation using the Newton Raphson method of partial differential correction Exp. Mech. 29 261-7

Chandra S and Shah S P 1968 Critical stress, volume change and microcracking of concrete ACI J. Proc. 65 770-80
Dongyu X, Chenga X, Huanga H and Jiang M 2010 Identifying technology for structural damage based on the impedance analysis of piezoelectric sensor Constr. Build. Mater. 24 $2522-7$

Dongyu X, Sourav B, Yanbing W, Shifeng H and Xin C 2014 Temperature and loading effects of embedded smart piezoelectric sensor for health monitoring of concrete structures Constr. Build. Mater. 76 187-93

Fuller C C, Sharon E and Nelson P A 1996 Active Control of Vibration (London: Academic)

Gopalaratnam V S and Shah S P 1985 Softening response of plain concrete in direct tension ACI J. Proc. 82 310-23

Liang C, Sun F P and Rogers C A 1994 An impedance method for dynamic analysis of active material systems J. Vib. Acoust. 116 $120-8$

Lim Y Y, Bhalla S and Soh C K 2006 Structural identification and damage diagnosis using self-sensing piezo-impedance transducers Smart Mater. Struct. 15 987-95

Lim Y Y and Soh C K 2012 Effect of varying axial load under fixed boundary condition on admittance signatures of electromechanical impedance technique J. Intell. Mater. Syst. Struct. (doi:10.1177/1045389X12437888)

Lim Y Y and Soh C K 2013 Damage detection and characterization using EMI technique under varying axial load Smart Struct. Syst. 11 349-64

Narayanan A and Subramaniam K V L 2016 Experimental evaluation of load-induced damage in concrete from distributed microcracks to localized cracking on electromechanical impedance response of bonded PZT Constr. Build. Mater. 105 536-44

Ong C W, Yang Y W, Naidu A S K, Lu Y and Soh C K 2002 Application of the electro-mechanical impedance method for the identification of in situ stress in structures Smart Structures, Devices, and Systems (Melbourne, December) ; Proc. SPIE 4935 503-14

Park G, Cudney H and Inman D 2000 Impedance-based health monitoring of civil structural components J. Infrastruct. Syst. 6 153-60

Saravanan T, Balamonica K, Priya C, Reddy A and Gopalakrishnan N 2015 Comparative performance of various smart aggregates during strength gain and damage states of concrete Smart Mater. Struct. 24085016

Schreier H and Sutton M 2002 Systematic errors in digital image correlation due to undermatched subset shape functions Exp. Mech. 42 303-10

Sirohi J and Chopra I 2000 Fundamental understanding of piezoelectric strain sensors J. Intell. Mater. Syst. Struct. 11 246-57

Soh C K and Bhalla S 2005 Calibration of piezo-impedance transducers for strength prediction and damage assessment of concrete Smart Mater. Struct. 14 671-84

Soh C K, Tseng K K H, Bhalla S and Gupta A 2000 Performance of smart piezoceramic patches in health monitoring of a $\mathrm{RC}$ bridge Smart Mater. Struct. 9 533-42

Tseng K K and Wang L 2004 Smart piezoelectric transducers for in situ health monitoring of concrete Smart Mater. Struct. 17 1017-24

VIC-2D ${ }^{\mathrm{TM}}$ Reference Manual 2009 Correlated Solutions

Wang D, Song H and Zhu H 2013 Numerical and experimental studies on damage detection of a concrete beam based on PZT admittances and correlation coefficient Constr. Build. Mater. $49564-74$

Xu Y G and Liu G R 2002 A modified electro-mechanical impedance model of piezoelectric actuator-sensors for debonding detection of composite patches J. Intell. Mater. Syst. Struct. 13 389-96

Yang Y and Hu Y 2008 Electromechanical impedance modeling of PZT transducers for health monitoring of cylindrical shell structures Smart Mater. Struct. 17 1-11 
Yang Y, Xu J and Soh C K 2005 Generic impedance-based model for structure-piezoceramic interacting system $J$. Aerospace

Eng. 18 93-101 